\title{
Divisibility Properties of the Fibonacci, Lucas, and Related Sequences
}

\author{
Thomas Jeffery and Rajesh Pereira \\ Department of Mathematics and Statistics, University of Guelph, Guelph, ON, Canada N1G 2W1 \\ Correspondence should be addressed to Rajesh Pereira; pereirar@uoguelph.ca
}

Received 13 December 2013; Accepted 22 January 2014; Published 4 March 2014

Academic Editors: H. Airault and W. Gao

Copyright (C) 2014 T. Jeffery and R. Pereira. This is an open access article distributed under the Creative Commons Attribution License, which permits unrestricted use, distribution, and reproduction in any medium, provided the original work is properly cited.

We use matrix techniques to give simple proofs of known divisibility properties of the Fibonacci, Lucas, generalized Lucas, and Gaussian Fibonacci numbers. Our derivations use the fact that products of diagonal matrices are diagonal together with Bezout's identity.

\section{Introduction}

The Fibonacci series is one of the most interesting series in mathematics. It is a two-term recurrence, where $F_{1}=F_{2}=1$ and $F_{n+1}=F_{n}+F_{n-1}$. The first few terms are $\{1,1,2,3,5$, $8,13,21,34,55\}$. The Lucas sequence is a related sequence with the same recurrence $L_{n+1}=L_{n}+L_{n-1}$ but different starting values of $L_{1}=1$ and $L_{2}=3$. The Fibonacci and Lucas sequences are special cases of the generalized Lucas sequences studied by Lucas in [1]. We will study these sequences in section two and the Gaussian Fibonacci sequences of Jordan [2] will be studied in section three. In this paper, we will give some easy matrix theoretical proofs of some well-known divisibility properties of these sequences. All of these proofs use the arithmetic of matrices over rings and two elementary ideas: Bezout's identity and the fact that any power of a diagonal matrix is a diagonal matrix. This gives an elementary and unified derivation of the divisibility properties of all of these sequences. We begin by reviewing some of the elementary terminologies of rings and properties of matrices over rings. We only assume that the reader is familiar with the definition of a principal ideal domain.

Definition 1. Let $R$ be a commutative ring with identity and let $a \in R$. Then $a$ is called a unit of $R$ if there exists $a^{-1} \in R$ such that $a a^{-1}=1$.
We will use two by two matrices over certain rings to give some easy proofs of some of the divisibility properties of these sequences. We will need the following result.

Proposition 2. Let $R$ be a commutative ring with identity and let $M=\left(\begin{array}{ll}a & b \\ c & d\end{array}\right)$ be a two by two matrix with entries in $R$. If the determinant of $M(a d-b c)$ is a unit, then $M$ is an invertible matrix.

In fact, the converse of this result is true as well and both of the original proof and the converse remain true for square matrices of arbitrary size.

Proof. If $a d-b c$ is invertible, then simple matrix multiplication shows us that $M^{-1}=(a d-b c)^{-1}\left(\begin{array}{cc}d & -b \\ -c & a\end{array}\right)$.

We now introduce the concept of the greatest common divisor and note some of its properties.

Definition 3. Let $R$ be a principal ideal domain and let $a, b \in$ $R$; then an element $c \in R$ is called the greatest common divisor of $a$ and $b$ (denoted by $\operatorname{gcd}(a, b)$ ) if $c$ is a divisor of both $a$ and $b$ and if any other common divisors of both $a$ and $b$ also divide $c$.

Proposition 4. Let $R$ be a principal ideal domain and let $a, b \in$ $R$. Then the greatest common divisor of $a$ and $b$ exists and is 
unique up to multiplication by a unit. Furthermore there exists $x, y \in R$ such that $x a+y b=\operatorname{gcd}(a, b)$.

Proof. Let $I$ be the ideal generated by $a$ and $b$. Then $I=$ $\{x a+y b: x, y \in R\}$. As $R$ is a principal ideal domain, $I$ is generated by a single element. This element is unique up to multiplication by a unit. Clearly the generator of $I$ satisfies both properties of the GCD and any GCD of $a$ and $b$ will be a generator of $I$.

This result is sometimes called Bezout's identity. We can use Bezout's identity to prove the following result which will be useful later on.

Proposition 5. Let $R$ be a principal ideal domain and let $a, b \in$ $R$. Do not let $b$ be a unit. Then $a$ is invertible in $R / b R$ (or equivalently $a$ is invertible $\bmod b)$ if and only if $\operatorname{gcd}(a, b)=1$.

Proof. If $\operatorname{gcd}(a, b)=1$, then there exists $x, y \in R$ such that $x a+y b=1$ which means that $a$ is invertible in $R / b R$. Conversely, if $a$ is invertible $\bmod b$, let $x$ be the inverse of $a$ in $R / b R$. Then $b$ divides $x a-1$ in $R$ and hence there exists $y \in R$ such that $x a+y b=1$ and $\operatorname{gcd}(a, b)=1$.

When $R=\mathbb{Z}, \bmod b$ is usually used to denote arithmetic in $\mathbb{Z} / b \mathbb{Z}$. We will also use the $\bmod b$ for arithmetic in $R / b R$ when $R$ is a general principal ideal domain. These are all the results in linear algebra and ring theory that we will need. The further theory of matrices over principal ideal domains as well as many other interesting topics in matrix theory can be found in [3].

\section{Divisibility Properties of Fibonacci and Lucas Numbers}

In this section, we give matrix theoretical proofs of the wellknown divisibility properties of the Fibonacci and Lucas numbers. Our proofs in this section use the well-known fact that $\left(\begin{array}{cc}F_{n+1} & F_{n} \\ F_{n} & F_{n-1}\end{array}\right)=\left(\begin{array}{ll}1 & 1 \\ 1 & 0\end{array}\right)^{n}$ which can easily be proven by induction. This identity forms the basis for one of the standard proofs of Cassini's identity $F_{n+1} F_{n-1}-F_{n}^{2}=(-1)^{n}$. We follow the usual convention by letting $Q$ denote the matrix $\left(\begin{array}{ll}1 & 1 \\ 1 & 0\end{array}\right)$. The $Q$ matrix has appeared in many proofs; see [4] for a detailed history of the $Q$ matrix. We note that $Q$ is an invertible matrix. As a demonstration of our methods, we provide a one-line proof of the following well-known divisibility property of the Fibonacci sequence.

Proposition 6. Let $k, n \in \mathbb{N}$; then $F_{n}$ divides $F_{k n}$.

Proof. $Q^{n}$ is a diagonal matrix mod $F_{n}$ which means that $Q^{n k}=\left(Q^{n}\right)^{k}$ is also a diagonal matrix $\bmod F_{n}$ and hence $F_{n}$ divides $F_{k n}$.

Theorem 7. For all $m, n \in \mathbb{N}, F_{g c d(m, n)}=\operatorname{gcd}\left(F_{m}, F_{n}\right)$.

Proof. It follows immediately from Proposition 6 that $F_{\operatorname{gcd}(m, n)}$ divides $\operatorname{gcd}\left(F_{m}, F_{n}\right)$. We now show that $\operatorname{gcd}\left(F_{m}, F_{n}\right)$ divides $F_{\operatorname{gcd}(m, n)}$. If $\operatorname{gcd}\left(F_{m}, F_{n}\right)=1$, we are done so suppose that $\operatorname{gcd}\left(F_{m}, F_{n}\right)>1$. Let $a$ and $b$ be integers such that $a m+b n=\operatorname{gcd}(m, n)$. Then $Q^{\operatorname{gcd}(m, n)}=\left(Q^{m}\right)^{a}\left(Q^{n}\right)^{b}$ (one of $a$ or $b$ will be negative which is not a problem since $Q$ is invertible and the inverse of a diagonal matrix is diagonal). Since $Q^{m}$ and $Q^{n}$ are diagonal mod $\operatorname{gcd}\left(F_{m}, F_{n}\right)$, so is $Q^{\operatorname{gcd}(m, n)}$. Hence $\operatorname{gcd}\left(F_{m}, F_{n}\right)$ divides $F_{\operatorname{gcd}(m, n)}$ which means that $F_{\operatorname{gcd}(m, n)}=\operatorname{gcd}\left(F_{m}, F_{n}\right)$.

We now derive similar results for the Lucas sequence. We note that if we let $A$ be the matrix $\left(\begin{array}{cc}1 & 2 \\ 2 & -1\end{array}\right)$, then $A Q^{n}=$ $\left(\begin{array}{cc}L_{n+1} & L_{n} \\ L_{n} & L_{n-1}\end{array}\right)$. We note that $A=2 Q-I$ and hence $A$ and $Q$ commute. Also note that $A^{2}=5 I$.

Proposition 8. Let $k, n \in \mathbb{N}$ with $k$ odd; then $L_{n}$ divides $L_{k n}$.

Proof. We begin by deriving the Cassini identity for the Lucas sequence. By taking determinants, we get $L_{n+1} L_{n-1}-L_{n}^{2}=$ $\operatorname{det}\left(A Q^{n}\right)=(-1)^{n+1} 5$. It follows that no element of the Lucas sequence is divisible by five as this would force all elements of the Lucas sequence to be divisible by five. Since $k$ is odd, let $k=2 m+1 . A Q^{n}$ is a diagonal matrix $\bmod L_{n}$ which means that $5^{m} A Q^{n k}=\left(A Q^{n}\right)^{k}$ is also a diagonal matrix $\bmod L_{n}$. Since $L_{n}$ is not divisible by five, $L_{n}$ divides $L_{k n}$.

A nearly identical argument gives us the following result.

Proposition 9. Let $k, n \in \mathbb{N}$ with $k$ even; then $L_{n}$ divides $F_{k n}$.

We also have a simple proof of the following.

Theorem 10. Let $m, n \in \mathbb{N}$ and let $d=\operatorname{gcd}(m, n)$. If $m / d$ and $n / d$ are both odd, then $\operatorname{gcd}\left(L_{m}, L_{n}\right)=L_{d}$.

Proof. It follows from Proposition 8 that $L_{d}$ divides $\operatorname{gcd}\left(L_{m}, L_{n}\right)$. We now show that $\operatorname{gcd}\left(L_{m}, L_{n}\right)$ divides $L_{d}$. If $\operatorname{gcd}\left(L_{m}, L_{n}\right)=1$, we are done so suppose that $\operatorname{gcd}\left(L_{m}, L_{n}\right)>1$. Since no element of the Lucas sequence is divisible by five and $\operatorname{det}(A)=-5, A$ must be invertible $\bmod L_{n}$ for any $n \in \mathbb{N}$. Let $a$ and $b$ be integers such that $a m+b n=d$. We note that one of $a$ to $b$ must be odd and the other must be even. $A Q^{m}$ and $A Q^{n}$ are both diagonal matrices $\bmod \operatorname{gcd}\left(L_{m}, L_{n}\right)$ and so is $\left(A Q^{m}\right)^{a}\left(A Q^{n}\right)^{b}$ which is equal to a power of five times $A Q^{d}$. Hence $\operatorname{gcd}\left(L_{m}, L_{n}\right)$ divides $L_{d}$ and $\operatorname{gcd}\left(L_{m}, L_{n}\right)=L_{d}$.

\section{Generalized Lucas Sequences}

In [1], Edouard Lucas investigated some useful sequences which have come to be known as the generalized Lucas sequences. We will show that the matrix methods of the previous section can be used to give some simple proofs of the divisibility properties of the generalized Lucas sequences. These divisibility properties can be found in Lucas' original paper [1] (see also [5] or chapter 1 of [6]).

Definition 11. Let $p$ and $q$ be integers; then the generalized Lucas sequence of the first kind $\left\{U_{n}(p, q)\right\}_{n=0}^{\infty}$ is the solution to the recurrence relation $U_{n+1}=p U_{n}-q U_{n-1}$ with initial conditions $U_{0}=0$ and $U_{1}=1$. The generalized Lucas 
sequences of the second kind $\left\{V_{n}(p, q)\right\}_{n=0}^{\infty}$ satisfy the exact same recurrence relation $V_{n+1}=p V_{n}-q V_{n-1}$ but have initial conditions of $V_{0}=2$ and $V_{1}=p$. The discriminant of either kind of generalized Lucas sequences is the quantity $\Delta=p^{2}-4 q$.

We are using lower case $p$ and $q$ instead of the more standard capital $P$ and $Q$ so as to avoid confusion with the $Q$ matrix. Many important integer sequences are a special case of the generalized Lucas sequence. Consider that $U_{n}(1,-1)$ are the Fibonacci numbers and $V_{n}(1,-1)$ are the Lucas sequence. Consider that $U_{n}(2,-1)$ are the Pell numbers; consider that $U_{n}(3,2)=2^{n}-1$ are the Mersenne numbers and $U_{n}(1,-2)$ are the Jacobsthal numbers and $V_{n}(1,-2)$ are the Jacobsthal-Lucas numbers. In all of these cases $p$ and $q$ are relatively prime and $\Delta=p^{2}-4 q$ is nonzero. We note that the only choices of $p$ and $q$ which are relatively prime and for which $\Delta=p^{2}-4 q=0$ are $(p, q)=(2,1)$ and $(p, q)=(-2,1)$. It can be verified that $U_{n}(2,1)=n, V_{n}(2,1)=2, U_{n}(-2,1)=$ $(-1)^{n+1} n$, and $U_{n}(-2,1)=(-1)^{n+1} 2$; the divisibility properties of these sequences are obvious and hence we are justified in restricting ourselves to the cases where $\Delta=p^{2}-4 q$ is nonzero.

The Fibonacci polynomials $\left\{F_{n}(x)\right\}_{n=1}^{\infty}$ satisfy the recurrence relation $F_{n+1}(x)=x F_{n}(x)+F_{n-1}(x)$ with initial polynomials $F_{0}(x)=0$ and $F_{1}(x)=1$. The Lucas polynomials $\left\{L_{n}(x)\right\}_{n=1}^{\infty}$ satisfy the same recurrence but have different initial polynomials $L_{0}(x)=2$ and $L_{1}(x)=x$. If we relax the condition that $p$ is an integer and allow $p$ to the polynomial $x$, we note that the Fibonacci polynomials are $\left\{U_{n}(x,-1)\right\}_{n=0}^{\infty}$ and the Lucas polynomials are $\left\{V_{n}(x,-1)\right\}_{n=0}^{\infty}$. Our methods in this section also apply to these polynomials and hence the conclusions of Proposition 12 and Theorem 13 apply also to the Fibonacci polynomials and the conclusions of Proposition 15 and Theorem 16 also apply to the Lucas polynomials.

We will show that, if $p$ and $q$ are relatively prime, $\left\{U_{n}(p, q)\right\}_{n=0}^{\infty}$ satisfies the same divisibility properties as the Fibonacci numbers. We will use the matrix identity $\left(\begin{array}{cc}U_{n+1} & -q U_{n} \\ U_{n} & -q U_{n-1}\end{array}\right)=\left(\begin{array}{cc}p & -q \\ 1 & 0\end{array}\right)^{n}$ which can easily be proven by induction. This identity first appears in [7]. In the remainder of this section, we let $M$ denote $\left(\begin{array}{cc}p & -q \\ 1 & 0\end{array}\right)$.

By replacing $Q$ with $M$ in Proposition 6, we get the following.

Proposition 12. Let $k, n \in \mathbb{N}$; then $U_{n}$ divides $U_{k n}$.

Proof. $M^{n}$ is a diagonal matrix mod $U_{n}$ which means that $M^{n k}=\left(M^{n}\right)^{k}$ is also a diagonal matrix $\bmod U_{n}$ and hence $U_{n}$ divides $U_{k n}$.

We can also prove a generalization of Theorem 7. M unlike $Q$ will not be invertible over $\mathbb{Z}$ unless $q= \pm 1$. However $M$ will be invertible $\bmod U_{n}$ if $p$ and $q$ are relatively prime. We show this using the fact that $M$ will be invertible $\bmod n$ if and only if $q$ and $n$ are relatively prime. It follows immediately from the difference equation that if $U_{n}$ is relatively prime to $q$, then $U_{n+1}$ is relatively prime to $q$. Therefore, by mathematical induction, $U_{n}$ is relatively prime to $q$ for all $n \geq 1$ which means that $M$ is invertible $\bmod U_{n}$ for all $n \geq 1$ when $p$ and $q$ are relatively prime. Now, by replacing $Q$ by $M$ in Theorem 7, we get a proof of the following result.

Theorem 13. Let $p$ and $q$ be relatively prime integers. Then for all $m, n \in \mathbb{N}, U_{\operatorname{gcd}(m, n)}=\operatorname{gcd}\left(U_{m}, U_{n}\right)$.

Proof. It follows immediately from Proposition 12 that $U_{\operatorname{gcd}(m, n)}$ divides $\operatorname{gcd}\left(U_{m}, U_{n}\right)$. We now show that $\operatorname{gcd}\left(U_{m}, U_{n}\right)$ divides $U_{\operatorname{gcd}(m, n)}$. If $\operatorname{gcd}\left(U_{m}, U_{n}\right)=1$, we are done so suppose that $\operatorname{gcd}\left(U_{m}, U_{n}\right)>1$. Let $a$ and $b$ be integers such that $a m+b n=\operatorname{gcd}(m, n)$. Then $M^{\operatorname{gcd}(m, n)}=\left(M^{m}\right)^{a}\left(M^{n}\right)^{b}$ is diagonal modgcd $\left(U_{m}, U_{n}\right)$. Since $M^{m}$ and $M^{n}$ are diagonal $\bmod \operatorname{gcd}\left(U_{m}, U_{n}\right)$, so is $M^{\operatorname{gcd}(m, n)}$. Hence $\operatorname{gcd}\left(U_{m}, U_{n}\right)$ divides $U_{\operatorname{gcd}(m, n)}$ which means that $U_{\operatorname{gcd}(m, n)}=\operatorname{gcd}\left(U_{m}, U_{n}\right)$.

We note that our proofs of Proposition 12 and Theorem 13 also work for the Fibonacci polynomials.

We also have a matrix identity for the generalized Lucas sequences of the second kind. Let $Z=\left(\begin{array}{cc}p-2 q \\ 2-p\end{array}\right)$; then $\left(\begin{array}{cc}V_{n+1} & -q V_{n} \\ V_{n} & -q V_{n-1}\end{array}\right)=Z M^{n}$. This identity also follows easily from induction. Taking the determinants of both sides and dividing by $-q$, we get Cassini's identity for these sequences $V_{n+1} V_{n-1}-V_{n}^{2}=\Delta q^{n-1}$. From this we obtain a useful lemma.

Lemma 14. Let $p$ and $q$ be relatively prime integers with $\Delta=$ $p^{2}-4 q \neq 0$. If $p$ is odd, then $\Delta$ must be relatively prime to $V_{n}$ for all $n$. If $p$ is even, then $\Delta$ is divisible by four; all of the $V_{n} s$ are even and $V_{n} / 2$ is relatively prime to $\Delta / 4$ for all $n$.

Proof. Suppose that $p$ is even. Now we prove the theorem by induction on $n$. The base $n=1$ case follows from the fact that $V_{1} / 2=p / 2$ which is relatively prime to $\Delta / 4$. The inductive step follows from dividing the Cassini identity by four to get $\left(\left(V_{n-1} / 2\right)\left(V_{n+1} / 2\right)\right)-\left(V_{n} / 2\right)^{2}=(\Delta / 4) q^{n-1}$. The case where $p$ is odd is proved similarly.

Note that $q$ is relatively prime to $V_{n}$ for all $n$ which means that $M$ is invertible $\bmod V_{n}$ for all $n$ (if $q$ shares a prime factor with one element of the sequence $\left\{V_{n}\right\}$, then by Cassini's identity every element of this sequence would be divisible by this factor including $p$ ). We note that $Z=2 M-p I$ and hence $M$ and $Z$ commute. Also note that $Z^{2}=\Delta I$. We can now prove the following by replacing $Q$ with $M$ and $A$ with $Z$ in the proof of Proposition 8 .

Proposition 15. Suppose that $p$ and $q$ are relatively prime integers. Let $k, n \in \mathbb{N}$ with $k$ being odd; then $V_{n}$ divides $V_{k n}$.

Proof. If $\left|V_{n}\right|=1$ then we are done, so suppose that $\left|V_{n}\right| \neq 1$. Since $k$ is odd, let $k=2 m+1$. Notice that $Z M^{n}$ is a diagonal matrix $\bmod V_{n}$ which means that $\Delta^{m} Z M^{n k}=\left(Z M^{n}\right)^{k}$ is also a diagonal matrix $\bmod V_{n}$. If $p$ is odd, then $V_{n}$ is relatively prime to $\Delta$, which means that $V_{n}$ divides $V_{k n}$. If $p$ is even, then $(1 / 2) Z M^{n}$ is a diagonal matrix $\bmod V_{n} / 2$ which means that $\left(1 / 2^{k}\right) \Delta^{m} Z M^{n k}=\left((1 / 2) Z M^{n}\right)^{k}$ is also a diagonal matrix $\bmod V_{n} / 2$. Since $V_{n} / 2$ is relatively prime to $\Delta / 4, V_{n} / 2$ divides $V_{k n} / 2$. 
By replacing $Q$ with $M$ and $A$ with $Z$ in the proof of Theorem 10, we have a simple proof of the following.

Theorem 16. Suppose that $p$ and $q$ are relatively prime integers and $m, n \in \mathbb{N}$. Let $d=\operatorname{gcd}(m, n)$. If $m / d$ and $n / d$ are both odd, then $\operatorname{gcd}\left(V_{m}, V_{n}\right)=V_{d}$.

Proof. If $\Delta=0$, then we must have $p=2$ and $q=1$ for $p$ and $q$ to be relatively prime integers. In this case $V_{n}=2$ for all $n$ and our result clearly holds. We now suppose that $\Delta$ is nonzero which means that the matrix $Z$ is invertible. It follows from Proposition 15 that $V_{d} \operatorname{divides} \operatorname{gcd}\left(V_{m}, V_{n}\right)$. We now show that $\operatorname{gcd}\left(V_{m}, V_{n}\right)$ divides $V_{d}$. If $\operatorname{gcd}\left(V_{m}, V_{n}\right)=1$, we are done, so suppose $\operatorname{gcd}\left(V_{m}, V_{n}\right)>1$. Let $a$ and $b$ be integers such that $a m+b n=d$. We note that one of $a$ to $b$ must be odd and the other must be even. Suppose that $p$ is odd; then $Z M^{m}$ and $Z M^{n}$ are both diagonal matrices $\bmod \operatorname{gcd}\left(V_{m}, V_{n}\right)$ and so is $\left(Z M^{m}\right)^{a}\left(Z M^{n}\right)^{b}$ which is equal to a power of $\Delta$ times $Z M^{d}$. Hence $\operatorname{gcd}\left(V_{m}, V_{n}\right)$ divides $V_{d}$ and $\operatorname{gcd}\left(V_{m}, V_{n}\right)=$ $V_{d}$. Now suppose that $p$ is even; then both $(1 / 2) Z M^{m}$ and $(1 / 2) Z M^{n}$ are diagonal matrices $\bmod \operatorname{gcd}\left(V_{m} / 2, V_{n} / 2\right)$. Since $(\Delta / 4)^{(a+b-1) / 2}\left((1 / 2) Z M^{d}\right)=\left((1 / 2) Z M^{m}\right)^{a}\left((1 / 2) Z M^{n}\right)^{b}$ and $\Delta / 4$ is relatively prime to $V_{n} / 2$ for all $n,(1 / 2) Z M^{d}$ is diagonal $\bmod \operatorname{gcd}\left(\left(V_{m} / 2\right),\left(V_{n} / 2\right)\right)$. Hence $\operatorname{gcd}\left(\left(V_{m} / 2\right),\left(V_{n} / 2\right)\right)$ divides $V_{d} / 2$ and $\operatorname{gcd}\left(V_{m}, V_{n}\right)=V_{d}$.

We can also use matrix techniques to prove some relations between the $U_{n}$ and the $V_{n}$ for fixed values of $p$ and $q$.

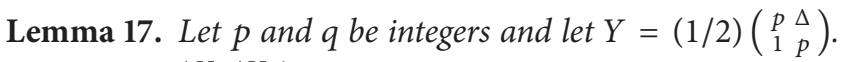
Then $2 Y^{n}=\left(\begin{array}{cc}V_{n} & \Delta U_{n} \\ U_{n} & V_{n}\end{array}\right)$.

Proof. We can easily verify that the result holds for $n=0$ and $n=1$. Since the trace of $Y$ is $p$ and the determinant of $Y$ is $q$, $Y$ has characteristic polynomial $x^{2}-p x+q$. By the CayleyHamilton theorem, $Y^{n+2}=p Y^{n+1}-q Y^{n}$ for all $n$; our result follows.

By taking the determinants of both sides of $2 Y^{n}=$ $\left(\begin{array}{cc}V_{n} & \Delta U_{n} \\ U_{n} & V_{n}\end{array}\right)$, we get the following identity.

Corollary 18. Let $p$ and $q$ be integers and let $\Delta=p^{2}-4 q$; then $V_{n}^{2}-\Delta U_{n}^{2}=4 q^{n}$.

\section{Gaussian Fibonacci Numbers}

All of the divisibility results in this section are new proofs of results in [2] where the Gaussian Fibonacci numbers were first defined. The Gaussian Fibonacci numbers are a sequence of Gaussian integers. The ring of Gaussian integers is the set of complex numbers whose real and imaginary parts are both integers. Consider that $\mathbb{Z}[i]=\{a+b i: a, b \in \mathbb{Z}\}$. The Gaussian integers form a unique factorization domain.

We follow [2] in defining the Gaussian Fibonacci numbers as follows. Let $f_{0}=i, f_{1}=1$, and $f_{n+1}=f_{n}+f_{n-1}$. It follows from induction that $f_{n}=F_{n}+i F_{n-1}$. We now let $X=$ $\left(\begin{array}{cc}1+i & 1 \\ 1 & i\end{array}\right)$. We note that $X^{2}=(1+2 i) Q$ and $X Q^{n-1}=\left(\begin{array}{cc}f_{n+1} & f_{n} \\ f_{n} & f_{n-1}\end{array}\right)$. By taking determinants of both sides of the latter equation, we get Cassini's formula for the Gaussian Fibonacci numbers $f_{n+1} f_{n-1}-f_{n}^{2}=(2-i)(-1)^{n}=(-i)(1+2 i)(-1)^{n}$. We note that $1+2 i$ is a Gaussian prime which divides the right-hand side of Cassini's formula and hence it is not a factor of any of the Gaussian Fibonacci numbers (if it were, it would be a factor of all of them including $f_{1}=1$, which is not the case). Since $\operatorname{det}(X)=-2+i=i(1+2 i), X$ is invertible $\bmod \operatorname{gcd}\left(f_{m}, f_{n}\right)$ for all $m, n \in \mathbb{N}$. It also follows from these two equations that $X^{2 n-1}=(1+2 i)^{n-1}\left(\begin{array}{cc}f_{n+1} & f_{n} \\ f_{n} & f_{n-1}\end{array}\right)$ for all $n \in \mathbb{N}$. We can now use these results to give new proofs of the divisibility properties of the Gaussian Fibonacci numbers.

Proposition 19. Let $k, n \in \mathbb{N}$. If $2 k-1$ divides $2 n-1$, then $f_{k}$ divides $f_{n}$.

Proof. $X^{2 k-1}$ is a diagonal matrix mod $f_{k}$. Therefore if $2 k-1$ divides $2 n-1$, then $X^{2 n-1}$ is also a diagonal matrix mod $f_{k}$ and hence $f_{k}$ divides $f_{n}$.

Theorem 20. Let $m, n \in \mathbb{N}$ and let $d$ be the natural number defined such that $2 d-1=\operatorname{gcd}(2 m-1,2 n-1)$; then $f_{d}=$ $\operatorname{gcd}\left(f_{m}, f_{n}\right)$.

Proof. It follows immediately from Proposition 19 that $f_{d}$ divides $\operatorname{gcd}\left(f_{m}, f_{n}\right)$. We now show that $\operatorname{gcd}\left(f_{m}, f_{n}\right)$ divides $f_{d}$. If $\left|\operatorname{gcd}\left(f_{m}, f_{n}\right)\right|=1$, we are done, so suppose that $\left|\operatorname{gcd}\left(f_{m}, f_{n}\right)\right|>1$. Let $a$ and $b$ be integers such that $a(2 m-$ $1)+b(2 n-1)=2 d-1$. Then $X^{2 d-1}=\left(X^{2 m-1}\right)^{a}\left(X^{2 n-1}\right)^{b}$. Since $X^{2 m-1}$ and $X^{2 n-1}$ are diagonal $\bmod \operatorname{gcd}\left(f_{m}, f_{n}\right)$, so is $X^{2 d-1}$. Hence $\operatorname{gcd}\left(f_{m}, f_{n}\right)$ divides $(1+2 i)^{d-1} f_{d}$. Since $(1+2 i)$ does not divide any Gaussian Fibonacci number, $\operatorname{gcd}\left(f_{m}, f_{n}\right)$ divides $f_{d}$ which means that $f_{\operatorname{gcd}(m, n)}=\operatorname{gcd}\left(f_{m}, f_{n}\right)$.

We note that we can introduce the following sequence with a parameter $\rho$ which contains as special cases the Fibonacci, Lucas, and the Gaussian Fibonacci sequences: $g_{n}=$ $F_{n}+\rho F_{n-1}$. When $\rho=0$ we get the ordinary Fibonacci sequence, when $\rho=2$ we get the Lucas sequence, and when $\rho=i$ we get the Gaussian Fibonacci sequence. We note that $\left(\begin{array}{cc}g_{n+1} & g_{n} \\ g_{n} & g_{n-1}\end{array}\right)=\left(I+\rho Q^{-1}\right) Q^{n}$. Taking the determinant of both sides, we get $g_{n+1} g_{n-1}-g_{n}^{2}=\left(1-\rho-\rho^{2}\right)(-1)^{n}$ which gives us the Cassini identity for all three of the sequences at once. If we take $\rho$ to be either $\rho_{1}=(-1+\sqrt{5}) / 2$ or $\rho_{2}=(-1-\sqrt{5}) / 2$, the right-hand side of the previous equation becomes zero and hence $\left\{F_{n}+\rho_{1} F_{n-1}\right\}$ and $\left\{F_{n}+\rho_{2} F_{n-1}\right\}$ are both geometric sequences. We note that $\left(I+\rho Q^{-1}\right)^{2}=\left(1+\rho^{2}\right) I+\left(2 \rho-\rho^{2}\right) Q^{-1}$. The values of $\rho$ which make either the $I$ term or the $Q^{-1}$ term on the right-hand side of this equation disappear are exactly the choices of $\rho$ which give us the Fibonacci sequence, the Lucas sequence, the Gaussian Fibonacci sequence, or the conjugate of the Gaussian Fibonacci sequence. This goes some way towards explaining why these sequences amongst those we get for other choices of $\rho$ have such nice divisibility properties.

\section{Conflict of Interests}

The authors declare that there is no conflict of interests regarding the publication of this paper. 


\section{Acknowledgment}

The research was supported by the Natural Sciences and Engineering Research Council of Canada Discovery Grant no. 400550 .

\section{References}

[1] E. Lucas, "Theorie des fonctions numeriques simplement periodiques," American Journal of Mathematics, vol. 1, no. 2, pp. 184196, 1878.

[2] J. H. Jordan, "Gaussian Fibonacci and Lucas numbers," The Fibonacci Quarterly, vol. 3, pp. 315-319, 1965.

[3] D. Serre, Matrices: Theory and Applications, Springer, New York, NY, USA, 2002.

[4] A. W. Gould, "A history of the Fibonacci Q-matrix and a higherdimensional problem," The Fibonacci Quarterly, vol. 19, pp. 250257, 1981.

[5] W. L. McDaniel, "The G.C.D. in Lucas sequences and Lehmer number sequences," The Fibonacci Quarterly, vol. 29, no. 1, pp. 24-29, 1991.

[6] P. Ribenboim, My Numbers, My Friends, Springer, New York, NY, USA, 2000.

[7] J. L. Brenner, "June meeting of the Pacific Northwest section. 1. Lucas' matrix," The American Mathematical Monthly, vol. 58, no. 3, pp. 220-221, 1951. 


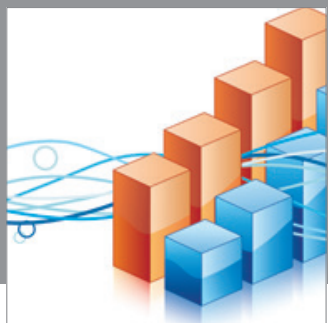

Advances in

Operations Research

mansans

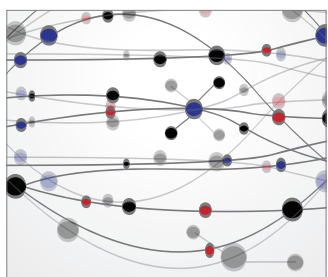

The Scientific World Journal
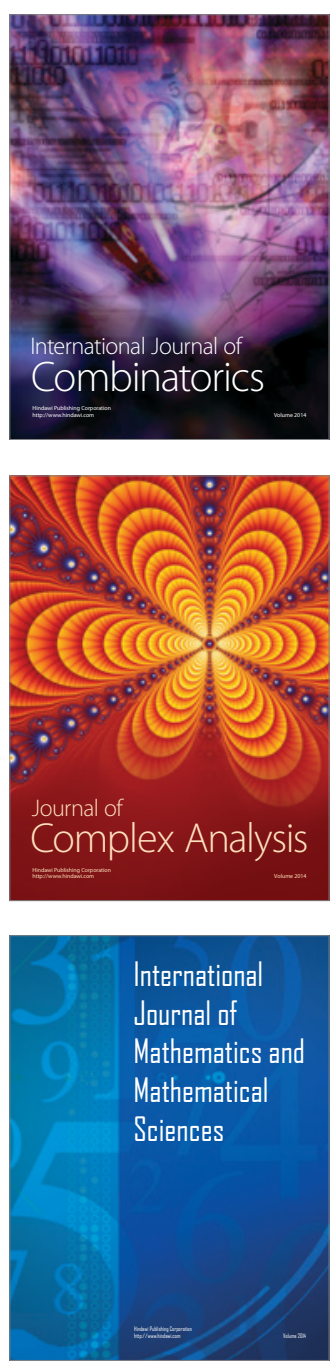
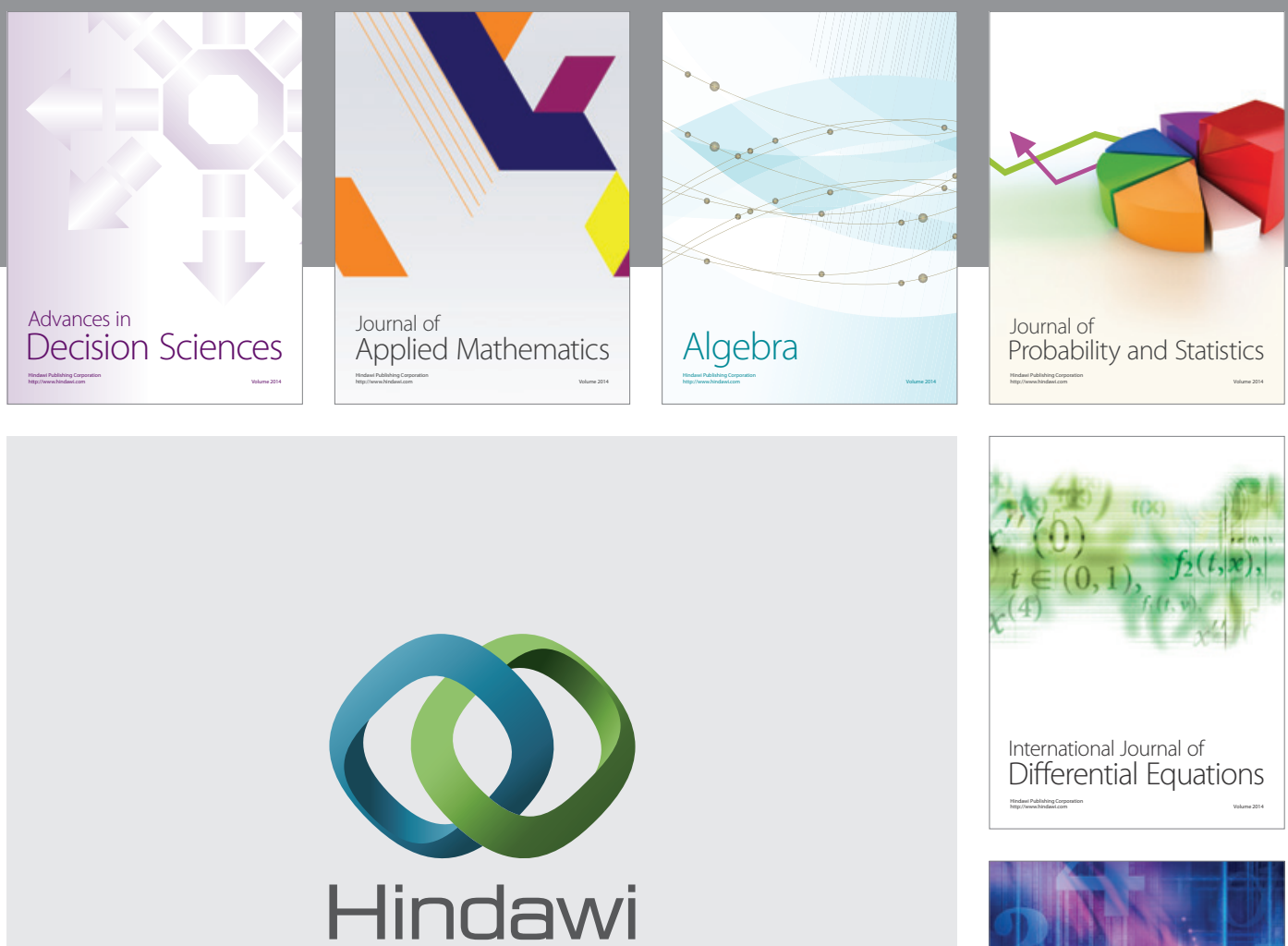

Submit your manuscripts at http://www.hindawi.com
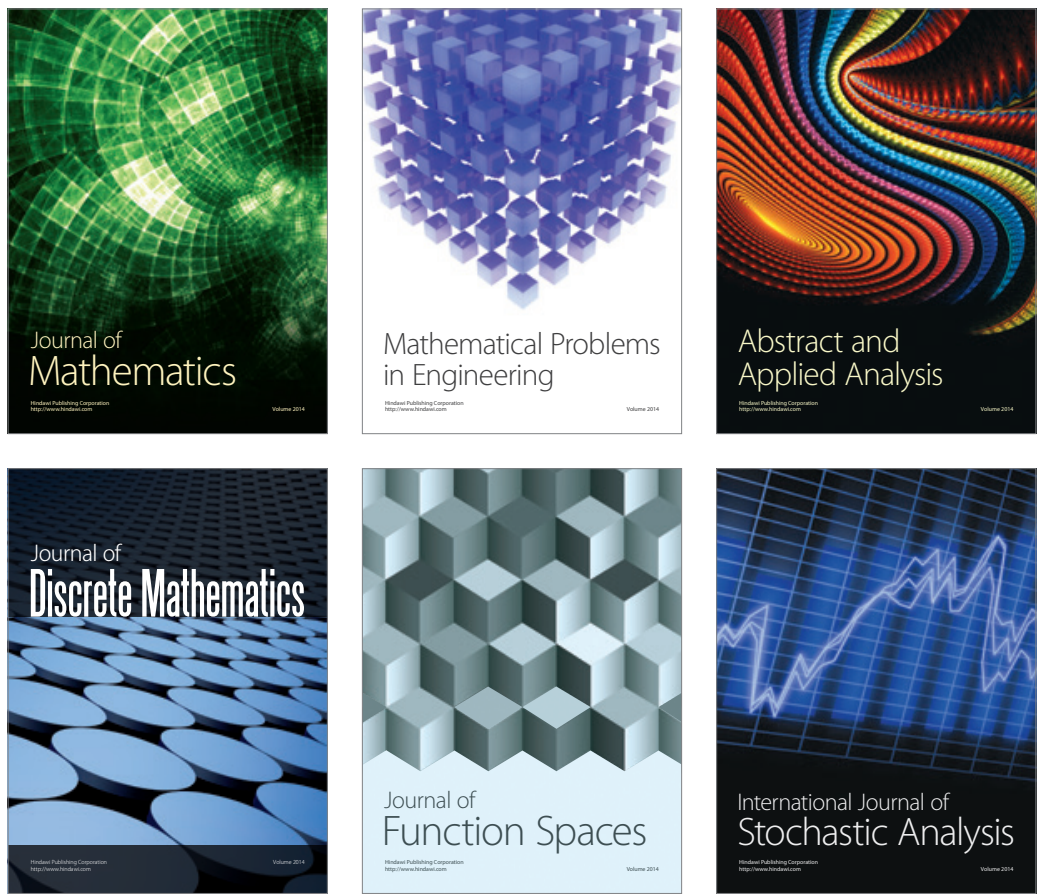

Journal of

Function Spaces

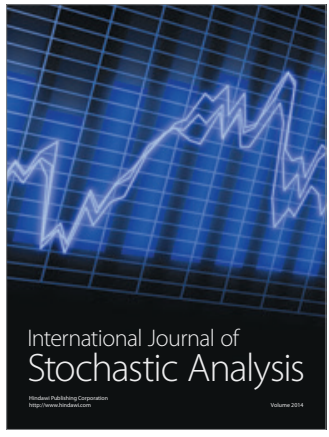

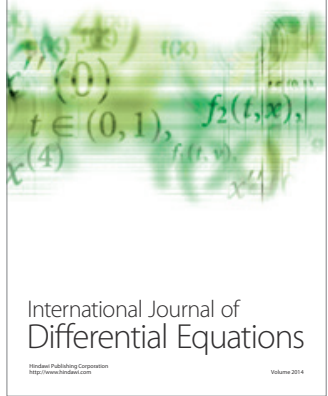
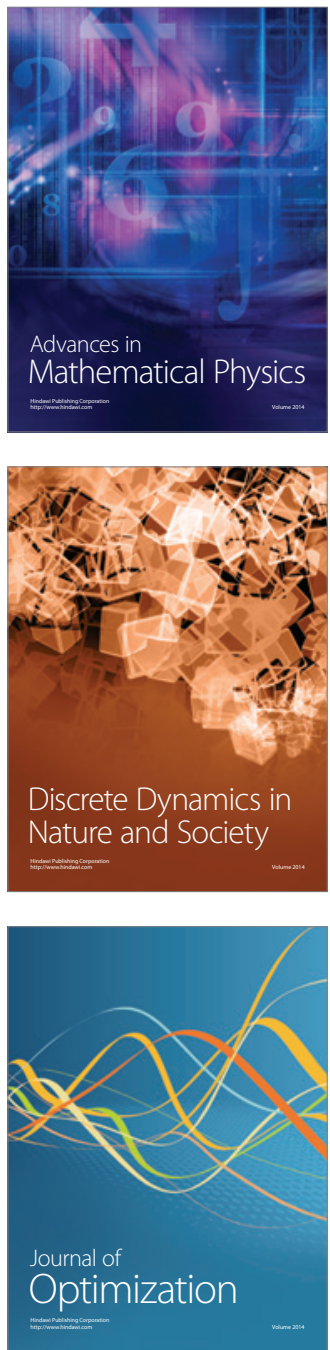